\title{
Estimation of Shelter Temperatures from Operational Satellite Sounder Data
}

\author{
P. A. DAVIS \\ Research and Data Systems, Incorporated, Lanham, MD 20706 \\ J. D. TARPLEY \\ National Earth Satellite Service, NOAA, Washington, DC 20233
}

(Manuscript received $1 \mathrm{July} 1982$, in final form 10 December 1982)

\begin{abstract}
As a first step in the development of a technique for estimating daily maximum and minimum shelter temperatures for agricultural monitoring, this study made use of operational satellite sounder data to estimate shelter temperature. Linear regression methods were used with a ground-truth data set of surface observations matched against data from operationally derived soundings. Regression estimates based solely on temperature predictors from the satellite soundings yielded residual standard deviations of 1.6-2.6 K for the NOAA-6 and NOAA-7 clear and partly cloudy retrievals. Regressions for cloudy conditions based only on the microwave retrievals had errors ranging from $2.9-4.0 \mathrm{~K}$. Shelter temperatures estimated by regression have errors somewhat smaller than those reported for the lower levels of the atmospheric soundings. This suggests that satellite temperature soundings near the surface are more accurate than previous studies indicate.
\end{abstract}

\section{Introduction}

There are several agricultural monitoring systems in operation whose function is to provide information on worldwide commodity production. These systems forecast crop production and provide alerts when abnormal weather events occur that affect crop conditions. A major problem in agricultural monitoring is the sparsity and untimeliness of ground-based meteorological observations. To alleviate this problem, there is an ongoing effort to use remotely sensed information to supplement conventional weather observations.

Daily maximum and minimum temperatures are important in crop yield models, soil moisture models, and crop stress detection. In the past, the only temperature data available to agricultural model developers have been observations of shelter temperature, from which daily temperature extremes were determined. Shelter temperature has a high spatial variability which contributes to crop monitoring errors in areas where observations are sparse and not representative of the whole region. Quantities derived from satellite data are by their nature area averages; for large crop districts, a single satellite estimate may be more representative than one or two conventional observations within the area.

The ability to monitor crop canopy temperature is a potential advantage of satellite observations. Canopy temperature (or skin temperature) is likely to be more directly related to crop growth and evapotranspiration than shelter temperature; the difference be- tween canopy temperature and shelter temperature is a measure of crop stress (Idso et al., 1977; Jackson et al., 1981). Satellite observations in the window regions of the infrared spectrum are closely related to canopy temperature, requiring only correction for atmospheric attenuation. Since crop models used currently were developed from shelter temperature, satellite-derived temperatures, to be useful, must also be expressed as equivalent shelter temperature.

The National Earth Satellite Service (NESS) has a program underway to develop techniques for estimating daily maximum and minimum temperatures from satellite data. The methods being developed are constrained to make use of data from currently operational satellites and be suitable for operational implementation. This paper reports on standard linear regression techniques to estimate shelter temperature from satellite sounder data. The second part of the research, to be reported later, is to use several satellite estimates of shelter temperature to determine daily maximum and minimum shelter temperatures.

The TIROS Operational Vertical Sounder (TOVS) system on the NOAA polar-orbiting satellites was chosen as the data source for shelter temperatures. The polar-orbiting satellites provide global coverage, as required for crop monitoring, while U.S. geostationary satellites only observe the Western Hemisphere. Two of the four daily observations of the NOAA satellites are at fortunate times for daily temperature extremes; one pass is in the early morning (0230 local time) near the expected minimum, and the other is in the afternoon (1430 local time) near 
expected maximum. Much effort has been expended on fully automated algorithms for obtaining clear radiances in the TOVS channels, and those procedures are part of the NESS operational sounding system (McMillin, 1978; McMillin and Dean, 1982).

\section{Coincident data sets}

Development of regression equations required a time and spatially coincident sounding and shelter temperature data set. To insure that any technique developed would be easily implemented into operation, the predictors in any regression equation were limited to quantities that are products of the NESS sounding system. This insures that the predictors will be relatively independent of TOVS calibration effects, filter response changes, changes in the sounding system, and future changes in the sounding resolution.

Atmospheric soundings at NESS are produced at a resolution ranging from 250 to $500 \mathrm{~km}$ depending on the nadir angle of the observations. The retrievals are derived from TOVS data arranged in "sounding boxes," whose dimensions are nine fields of view (FOV) along a scan line and seven scan lines along the satellite track. Six sounding boxes are acquired across the track: three on each side of the satellite subpoint. Relative to the satellite track, each set of six sounding boxes consists of inner, middle, and outer pairs of soundings that are associated with increasing scan angle. The size of each FOV is $20 \mathrm{~km}$ in the infrared sounder (High Resolution Infrared Sounder, HIRS); the microwave data (Microwave Sounding Unit, MSU) have a FOV of $110 \mathrm{~km}$ but, for processing, are analyzed to the HIRS resolution.

As the NESS sounding operation is currently configured, there are three possible ways each sounding box can be processed. Initially, each HIRS sample is tested to determine the probable presence of clouds. If as few as four completely cloud-free samples are found in a box, these values are used in the retrieval without further processing. If fewer than four clear FOV's are found, clear radiances are derived from partially cloud-filled samples. Profiles based on derived clear radiances are called partly cloudy retrievals. If an insufficient number of derived clear radiances are available, then retrievals designated as cloudy are made from only the microwave and highlevel HIRS channels and are designated as cloudy retrievals. About 20 percent or less of the profiles are retrieved using the microwave algorithms. The location assigned to each temperature sounding is the centroid of the FOV's used in the retrieval. A detailed description of the NESS atmospheric sounding system and further references to the physics and mathematics of satellite sounding can be found in NOAA Technical Report NESS 83, "Atmospheric Sounding User's Guide" (Werbowetzki, 1981) and a paper by Smith et al. (1979).
For this study, coincident data were collected over the area of North America between 25 and $60^{\circ} \mathrm{N}$ latitude and 70 and $105^{\circ} \mathrm{W}$ longitude. Data collection began in early April 1981 from NOAA-6 soundings. The equator crossing times for NOAA- 6 are 0730 and 1930 local times. Data from NOAA-7, with equator crossings at 0230 and 1430 local time, became available in late August 1981. Table 1 contains a list of selected satellite sounder products collected from the operational data stream and included in the data set. The skin temperature is the equivalent brightness temperature in the $11.1 \mu \mathrm{m}$ window channel corrected for atmospheric absorption; it therefore is interpreted as the skin or canopy temperature.

The National Meteorological Center (NMC) computer files of hourly surface observations were searched for all stations within a specified distance and time of each satellite retrieval. A case was included in the coincident data set if there were at least five stations reporting hourly temperature observations within 1 hour of retrieval time and located within $1.5^{\circ}$ of latitude and longitude of the retrieval centroid. The temperature at each station was corrected to the TOVS retrieval elevation by assuming a lapse rate of $0.56^{\circ} \mathrm{C}$ $\left(1.0^{\circ} \mathrm{F}\right)$ for every $100 \mathrm{~m}$ of elevation. Quantities taken from the surface reports and retained in the matched data set are listed in Table 2.

If fewer than two of the stations report cloud amount, the matchup was not used. It was necessary to have observations of cloud amount to avoid comparing surface temperatures from completely overcast stations with retrievals that are made from partially clear or clear FOV's. The number of exclusions with cloudiness reported between 0.5 and 0.75 in the vicinity of clear retrievals is high and illustrates the apparent success of the sounding algorithm in finding "holes" in the cloud cover. Identity of the stations was not maintained in the matched data set after temporal interpolation, height adjustment, and averaging. Likewise, in the operational temperature retrieval

TABLE 1. Elements of satellite sounder products included in surface-TOVS coincident data set.

Satellite identification number; date; time

Retrieval box number

Latitude and longitude, retrieval centroid location

Retrieval mode (clear, partly cloudy, cloudy)

Flags (land/sea; day/night; surface elevation)

Solar zenith angle

Retrieved atmospheric temperature at the highest 20 TOVS pressure levels

Geopotential heights; water vapor mixing ratios

Clear column brightness temperatures for all TOVS channels

Skin temperature

Surface height

Highest 20 TOVS pressure levels (mb)

$115,135,150,200,250,300,350,400,430,475,500,570,620$, $670,700,785,850,920,950,1000$ 
TABLE 2. Entries in surface-TOVS coincident data set from pairs of hourly surface observations.
Number of reports
Number of reports with cloud amount
Average cloud amount
Mean elevation $(\mathrm{m})$ for all stations used in matchup
Mean interpolated and elevation-adjusted temperature
Mean interpolated dewpoint temperature
Mean interpolated wind speed
Standard deviations of elevation, temperature, dewpoint, windspeed
Wind direction histogram (calm and four quadrants)

system, the location of FOV's used in the retrieval is not maintained once the centroid location is computed.

The standard deviation of the five or more shelter temperatures for each coincident matchup is a measure of variability inherent in shelter temperature. The average of all standard deviations for each matchup, denoted as $\bar{\sigma}_{\mathrm{SH}}$, is used as a quality standard for retrieved shelter temperatures.

\section{Regression model}

The different thermal structures of the lower atmosphere at times of maximum and minimum temperature are associated with different heat transport mechanisms. Fig. 1 illustrates expected near surface temperature profiles at the diurnal extremes. At night, increased stability tends to restrict the turbulent vertical exchange processes. Net loss of heat by radiation from the surface on clear nights causes temperature inversions and surface temperatures significantly lower than those of the overlying atmosphere. In addition, temperature variations caused by cold air drainage may result in lower local shelter temperatures and variable spatial distribution. During the day, surface heating may lead to shelter temperatures that are warmer than the lapse rate in the overlying atmosphere would suggest. Minimum temperatures typically deviate most from a direct extrapolation of retrieved atmospheric temperature, while maximum temperatures are determined in more regular fashion by convective mixing. A successful regression scheme must account for these diurnal variations in shelter temperature using TOVS products as predictors.

A simple linear regression approach was selected as a means for relating satellite-derived quantities to observed shelter temperatures. If $T_{\mathrm{SH}}$ is the dependent variable and $X_{i}$ represents independent variables selected from the TOVS products, observed values of $T_{\mathrm{SH}}$ are used with retrieval data products to determine regression coefficients $a_{i}$ from

$$
T_{\mathrm{SH}}(\mathrm{obs})=a_{0}+a_{1} X_{1}+a_{2} X_{2}+a_{3} X_{3}+\cdots .
$$

Criteria for determining the usefulness of a given set of independent variables as predictors of shelter temperature were 1) amount of explained variance, 2) reduction of the standard deviation of the residual toward the average standard deviation of the coincident shelter temperatures, 3) statistical significance of the regression coefficients, 4) accurate estimation of shelter temperature with independent data, and 5) convenience of operational implementation. The fifth criterion, operational convenience, biased the evaluation toward satellite-derived predictors, although the coincident data set contained quantities such as winds and surface dewpoint temperature that were useful in the regression. The data were stratified by time of day, retrieval type, etc., to allow the regression equation to more accurately reflect the physics of the situation.

An independent variable that proved useful in many regression equations was the extrapolated surface temperature, $T_{E}$. The extrapolation was done assuming a linear variation of temperature with the logarithm of pressure

$$
T_{E}=T_{1}+\left(T_{1}-T_{2}\right)\left(\ln p_{s}-\ln p_{1}\right) /\left(\ln p_{1}-\ln p_{2}\right),
$$

where $p_{1}, p_{2}, T_{1}$, and $T_{2}$ are the pressures and retrieved temperatures at the standard TOVS levels directly above the surface, and $p_{s}$ is the surface pressure. Surface pressure was estimated from the equation

$$
\ln p_{s}=\ln p_{0}-\left(Z_{s} / k T_{v}\right)
$$

where $p_{0}$ is standard sea level pressure $(1013.25 \mathrm{mb})$, $Z_{s}$ is the elevation of the surface (provided from the temperature retrieval), $k$ is a constant [29.3 for $T_{v}$ $(\mathrm{K})$ and height $(\mathrm{m})]$ and $T_{v}$ is an effective mean virtual temperature between sea level and the surface elevation. Some improvement in results might be achieved by allowing for seasonal and latitudinal changes in the reference level pressure. This procedure uses surface elevation to estimate mean surface pressure, which is then used in (2) for computing $T_{E}$.

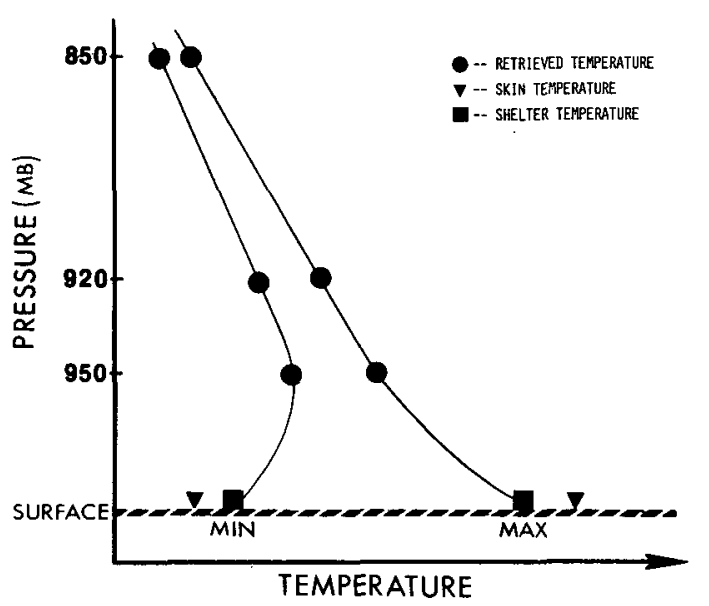

FIG. 1. Expected boundary region temperature distribution near daily maximum and minimum temperature, for clear conditions. 
4. Shelter temperature estimates from regression analysis

\section{a. Clear and partly cloudy retrievals, NOAA-6}

The first data set collected was NOAA- 6 retrievals for the period of April through July 1981. A total of 974 matchups at local solar times of around 0730 and 1930 were obtained. For these 4 months at the latitudes where the data were collected, the morning pass was after sunrise and the evening observation within an hour and a half of sunset, so the expected temperature profiles were well within the extreme cases illustrated in Fig. 1.

Initial analyses were for the clear and partly cloudy retrievals or their combination. Repeated stepwise regression analysis isolated and ordered the most significant variables. Wind speed, contrary to expectation, failed as a predictor of shelter temperature. Some of the surface observations, such as dewpoint, improved the regression relationship slightly; but, for ease in implementing the technique into an operation, our preference was to use only satellite sounder data.

Extensive analyses of variables with the 4-month NOAA-6 data set indicated that reasonable results could be obtained with only two independent variables taken from TOVS retrievals. Four or more variables sometimes lead to a poorer relationship, especially when applied to independent data. The two most significant variables for the morning-evening times during the growing season were found to be $T_{E}$ and $M_{2}$, the microwave channel 2 brightness temperature. Table 3 summarizes results for the 4-month data set of NOAA- 6 with a stratification by retrieval mode. Included in the table is the average standard deviation of the corresponding observed shelter temperatures, $\sigma_{\mathrm{SH}}$, which served as a standard for $\sigma_{R}$, the

TABLE 3. Summary of statistics from regression estimation of shelter temperatures using two independent variables $\left(T_{E}\right.$ and $\left.M_{2}\right)$ from NOAA-6, April-July 1981.

\begin{tabular}{|c|c|c|c|c|c|}
\hline \multicolumn{5}{|c|}{$\begin{array}{l}\text { Number of samples } \\
\text { Explained variance } \\
\text { Standard deviations of residuals } \\
\text { Average standard deviation of matched shelter } \\
\text { temperatures }\end{array}$} & \multirow[b]{2}{*}{$\sigma_{\mathrm{SH}}$} \\
\hline & Mode & $N$ & $R^{2}$ & $\sigma_{R}$ & \\
\hline April-July & $\begin{array}{l}\text { Clear } \\
\text { Partly cloudy } \\
\text { Combined }\end{array}$ & $\begin{array}{l}823 \\
151 \\
974\end{array}$ & $\begin{array}{l}0.94 \\
0.92 \\
0.94\end{array}$ & $\begin{array}{l}1.64 \\
2.07 \\
1.75\end{array}$ & $\begin{array}{l}1.49 \\
1.64 \\
1.51\end{array}$ \\
\hline April, May & $\begin{array}{l}\text { Clear } \\
\text { Partly cloudy } \\
\text { Combined }\end{array}$ & $\begin{array}{r}380 \\
69 \\
449\end{array}$ & $\begin{array}{l}0.92 \\
0.88 \\
0.90\end{array}$ & $\begin{array}{l}1.78 \\
2.57 \\
1.98\end{array}$ & $\begin{array}{l}1.53 \\
1.81 \\
1.57\end{array}$ \\
\hline June, July & $\begin{array}{l}\text { Clear } \\
\text { Partly cloudy } \\
\text { Combined }\end{array}$ & $\begin{array}{r}443 \\
82 \\
525\end{array}$ & $\begin{array}{l}0.86 \\
0.83 \\
0.85\end{array}$ & $\begin{array}{l}1.49 \\
1.39 \\
1.51\end{array}$ & $\begin{array}{l}1.46 \\
1.49 \\
1.46\end{array}$ \\
\hline
\end{tabular}

standard deviation of the difference between estimated temperature and $T_{\mathrm{SH}}$. The sample size and variance in the observed temperature explained by the regression are designated by $N$ and $R^{2}$, respectively, in the tables. The result in Table 3 establishes a baseline for retrieval accuracy attainable from a polar orbiter with morning and early evening overflights. On the average, performance is worse for the partly cloudy retrieval as expected.

Scatter diagrams of estimated and observed shelter temperatures for each retrieval mode and their combination are illustrated in Figs. $2 a, 2 b$, and $2 c$. The scatter diagrams show that bias about the mean is not pronounced.

When the data are separated into spring and summer, it is apparent that the greater explained variance but larger standard deviation of residuals occurs in spring. Average standard deviations of shelter temperatures in spring also exceeded those in summer. An interesting distinction between the spring and summer statistics is the poorer performance of partly cloudy retrievals compared with clear ones in spring, but with the reverse relationship in summer. Nevertheless, estimates are not significantly improved by the introduction of separate regression coefficients for spring and summer.

Another stratification of data, by retrieval-box scan angle, showed a deterioration in estimation accuracy for the outer boxes (largest scan angles). The reduced accuracy was caused by cloudiness and the greater screening of the surface by clouds when viewed from large nadir angles.

For a check on the application of regression coefficients to independent data, the 4-month NOAA-6 data set was divided into two portions with 488 matchups of dependent data interleaved with 486 matchups of independent data. Coefficients were derived from the dependent data set and were applied to the independent data to estimate shelter temperature. Results are summarized in Table 4. Again, of the independent variables tried, the two variables found to perform best during the period were $T_{E}$ and $M_{2}$. It is apparent from Table 4 that the two variables, when tested on independent data, are sufficient and provide estimates with standard deviations close to those in the dependent data. Explained variances for the independent data are several percent smaller. Scatterdiagrams of estimated and observed shelter temperatures for the independent data test appear in Fig. 3. They are similar to those based on dependent data in Fig. 2c.

\section{b. Cloudy retrievals, NOAA-6}

Data for the cloudy retrievals were treated in a separate regression analysis. For this mode, the tropospheric retrieved profile is based on microwave sounder data only. During the period April-August 

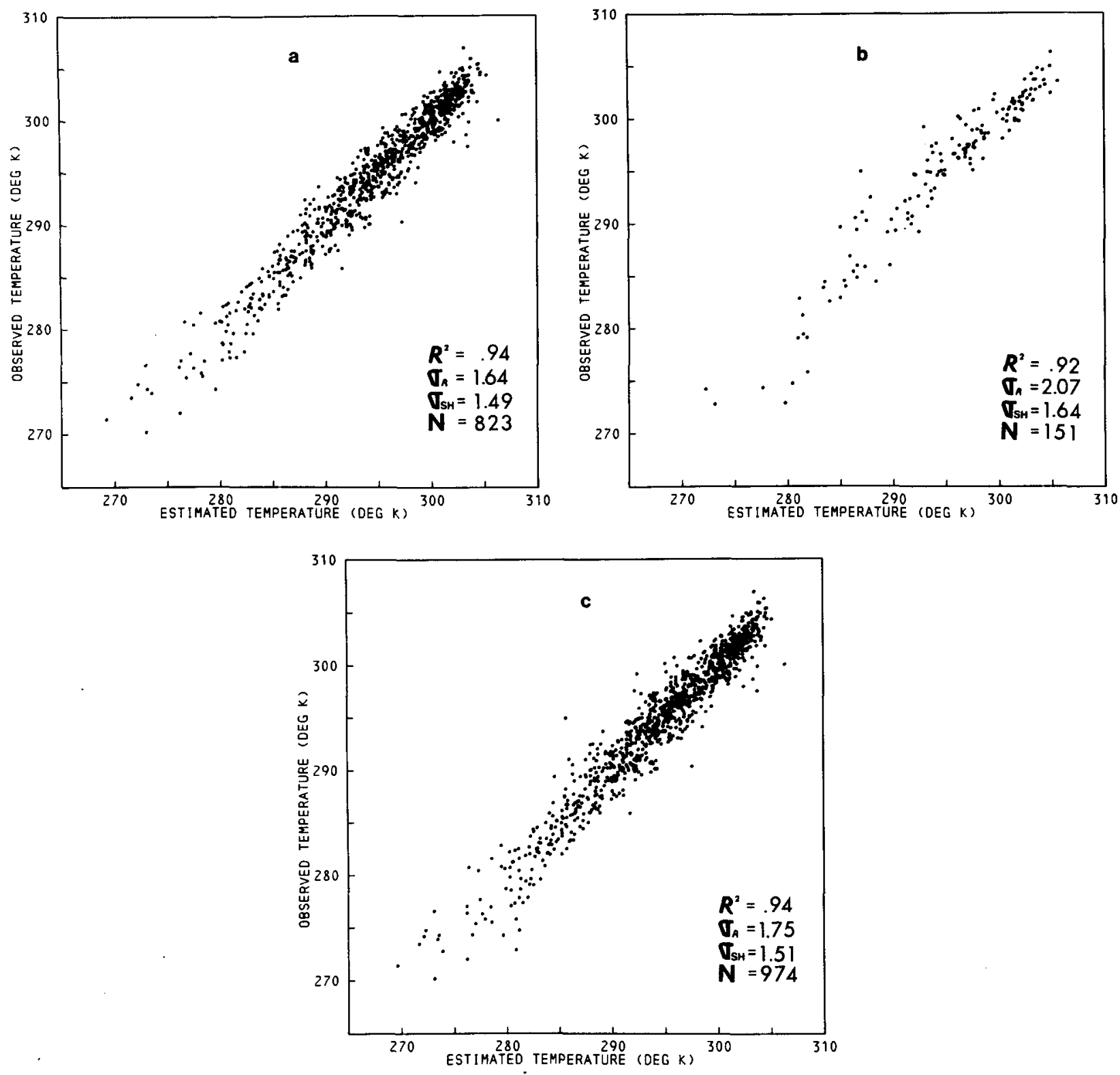

FIG. 2. Mean shelter temperature plotted against NOAA-6 satellite estimates (dependent data) from (a) clear TOVS retrievals, (b) partly cloudy TOVS retrievals and (c) both, for April through July 1981. The observed temperatures are averages of five or more interpolated, elevation-corrected shelter observations.

1981 , there were 203 cloudy data matchups. In the regression analysis, the same two variables, $T_{E}$ and $M_{2}$, that were used for the previous retrieval modes gave the results summarized in Table 5 . Overall performance is inferior to that of clear and partly cloudy retrievals, but was expected because of the lack of low-level sounding channels in the MSU and poorer spatial resolution. Evening results and ground-truth noise are worse than those for the morning. As before, the $R^{2}$ decreased and $\sigma_{R}$ increased for the outer scan boxes in comparison with corresponding statistics for inner boxes. The greater relative frequency of occur- rence of outer box retrievals probably results from failure of the TOVS clear radiance procedures because apparent cloudiness increases with scan angle.

The resultant scatterdiagram of estimated and observed temperatures for the cloudy retrievals (microwave only) is illustrated in Fig. 4. Although the scatter of data is larger than for other retrieval modes, there is no pronounced bias.

\section{c. Clear and partly cloudy retrievals, NOAA-7}

Initial analyses of NOAA- 6 data covered most of the growing season for the two local times of day. 
TABLE 4. Regression estimation results from selected dependent data with tests on independent data.

\begin{tabular}{llrrrr}
\hline \hline & \multicolumn{1}{c}{ Mode } & $N$ & $\sigma_{\text {SH }}$ & $R^{2}$ & $\sigma_{R}$ \\
\hline a. Dependent & Clear & 404 & 1.55 & 0.95 & 1.61 \\
$\quad$ data & Partly cloudy & 84 & 1.66 & 0.93 & 2.11 \\
$\quad$ (April-July) & Combined & 488 & 1.57 & 0.95 & 1.73 \\
b. Independent & Clear & 419 & 1.43 & 0.93 & 1.67 \\
$\quad$ data $^{*}$ & Partly cloudy & 67 & 1.61 & 0.91 & 2.10 \\
& Combined & 486 & 1.46 & 0.92 & 1.78 \\
\hline
\end{tabular}

* Using coefficients from all four months of dependent data.

With the advent of NOAA-7 data in late August 1981 , it became possible to examine the effect of thermal structures at other local times on the retrieval products. A NOAA-7 test data set was formed for data during the 1981 summer-fall transition. Using the same two independent variables, $T_{E}$ and $M_{2}$, that were used in the previous NOAA- 6 analyses, a regression analysis was performed without separation of data by day (D) or night (N). Fig. 5a illustrates results which are associated with an explained variance of only about $73 \%$ and a standard deviation of residuals of $4.25 \mathrm{~K}$. It is obvious that daytime estimates are too low and nighttime estimates are too high. A simple stratification of the data by day-night, with separate regressions, leads to the much improved results illustrated in Fig. 5b.

The two distributions of Figs. 5a and b correspond to the differences in surface layer stability found near minimum and maximum temperatures. For overestimates near minimum temperature and underesti-

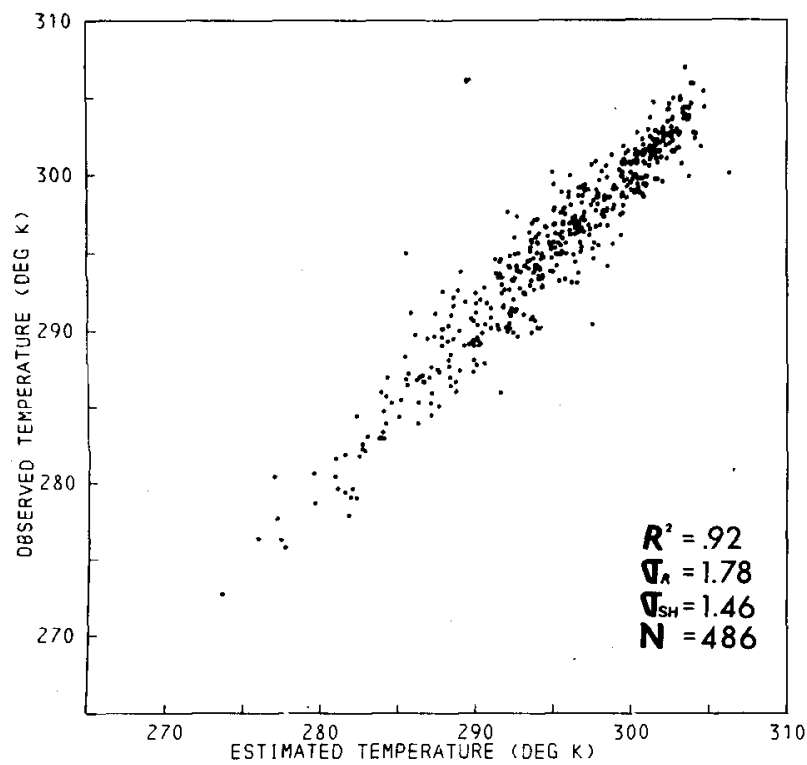

FiG. 3. Mean shelter temperatures plotted against NOAA-6 satellite estimates (independent data) from clear and partly cloudy retrievals for April through July 1981.
TABLE 5. Regression estimation results for NOAA-6 cloudy retrievals from April to August 1981 using two independent variables, $T_{E}$ and $M_{2}$.

\begin{tabular}{lrccc}
\hline \multicolumn{1}{c}{ Class } & $N$ & $R^{2}$ & $\sigma_{R}$ & $\sigma_{\mathrm{SH}}$ \\
\hline All data & 203 & 0.81 & 2.91 & 1.78 \\
Morning & 112 & 0.82 & 2.66 & 1.62 \\
Evening & 91 & 0.79 & 3.13 & 1.98 \\
Inner scans & 99 & 0.86 & 2.55 & 1.92 \\
Outer scans & 104 & 0.76 & 3.21 & 1.65 \\
\hline
\end{tabular}

mates near maximum temperature, the skin temperatures were closer to the shelter temperature than were values of $T_{E}$. Note that the average standard deviation of shelter temperature used in the matchup is significantly larger during the night than during the day. The stable conditions at night along with cold air drainage causes larger spatial variations in air temperature. As a result, regression estimations for clear nights (without additional stratification) are inferior to those for daytime. Illustrated results emphasize the importance of stratifying the data according to the thermal structure.

The main distinction then, between the NOAA- 6 and NOAA-7 data, appears to be the fact that NOAA7 observations occur near the two diurnal extremes in temperature whereas the NOAA- 6 observations are approximately equally spaced in time relative to time of maximum temperature. After sorting the NOAA7 data by day and night, an attempt was made to improve the estimates by considering additional or alternative independent variables or by additional sorting.

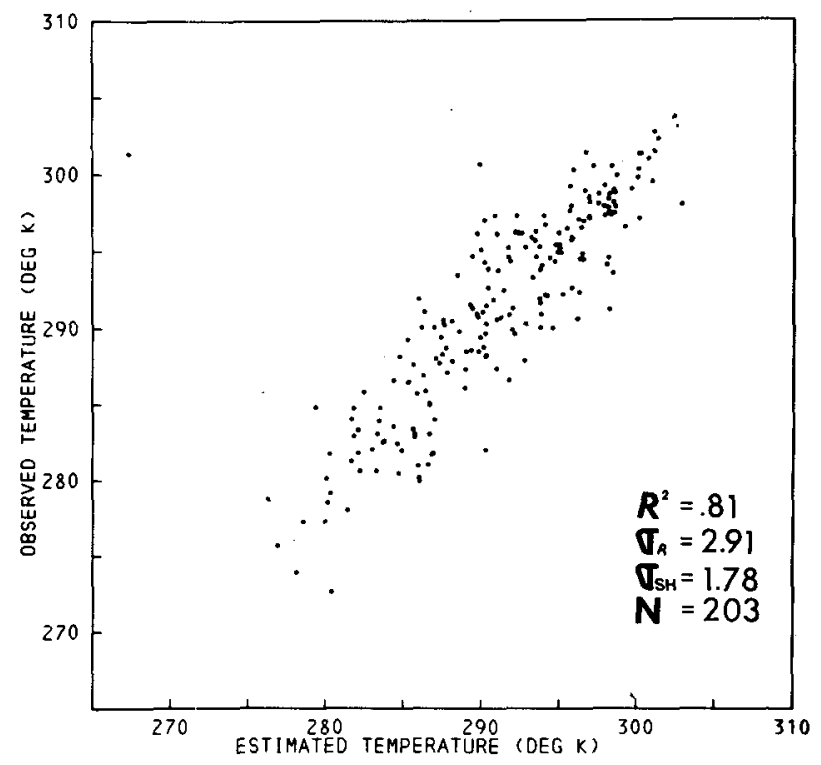

FIG. 4. Mean shelter temperatures plotted against NOAA-6 satellite estimates (dependent data) from microwave retrievals. 

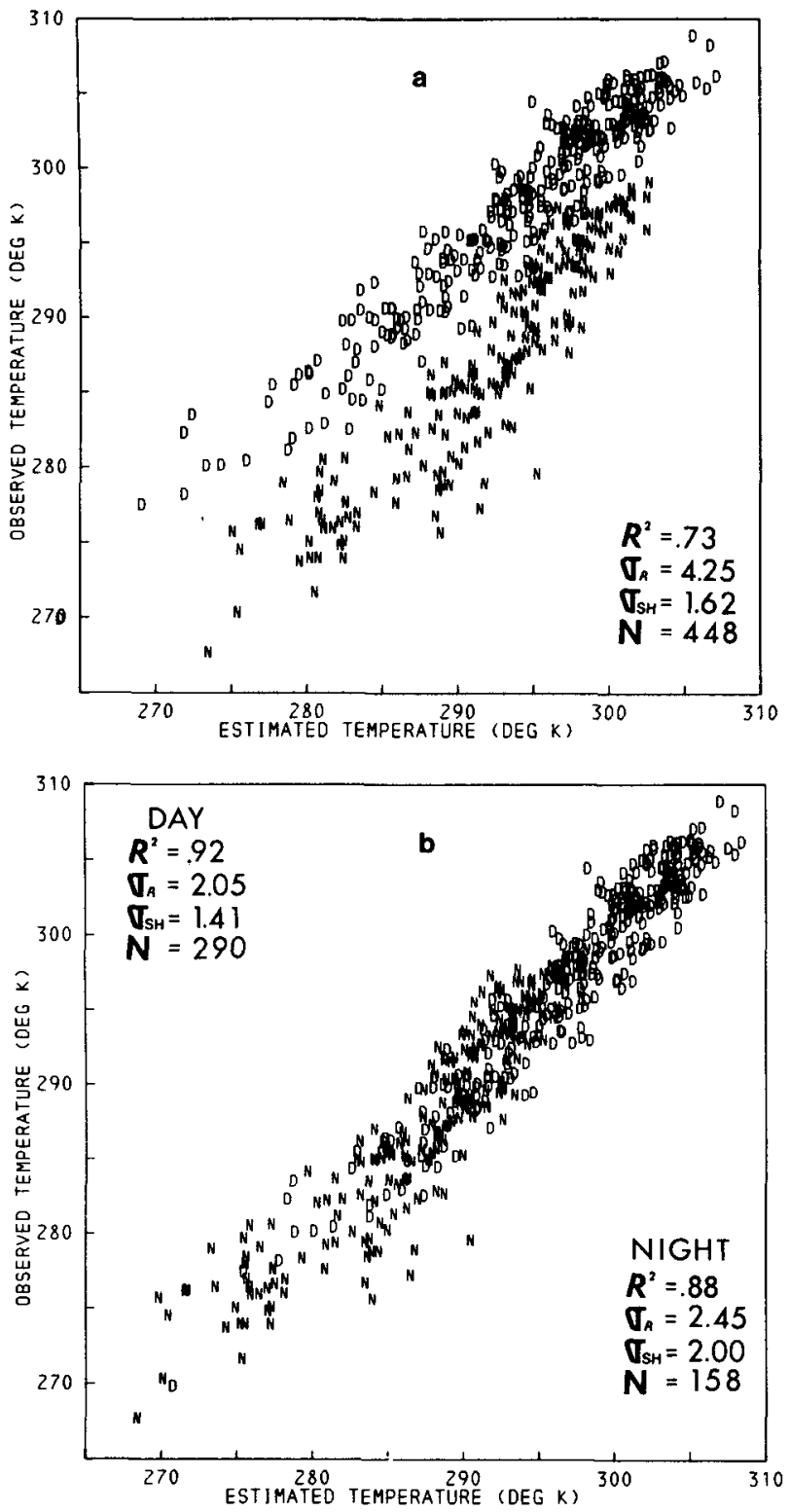

FIG. 5. Shelter temperatures plotted against NOAA-7 satellite estimates from (a) a single regression equation and (b) separate regressions for day and night. Daytime and nighttime observations are denoted as $\mathrm{D}$ and $\mathrm{N}$, respectively.

\section{Extended analyses}

\section{a. Data stratifications and the utility of regression}

Extension of the regression analyses to all seasons required use of the skin temperature, $T_{\mathrm{SK}}$, as a third independent variable,

$$
T_{\mathrm{SH}}=a_{0}+a_{1} T_{E}+a_{2} M_{2}+a_{3} T_{\mathrm{SK}} .
$$

Assessment of impacts of diurnal or seasonal variations in thermal structure were aided by subsequent substratifications of data according to the difference,
$\Delta T$, between the skin temperature and the lowest retrieval temperature $T_{\mathrm{RET}}$ :

$$
\Delta T=T_{\mathrm{SK}}-T_{\mathrm{RET}} .
$$

Initial stratification of NOAA- 6 data was by season, with April, May, June, July, August, and September denoted by S-S (spring, summer) and October, November, December, January, February, and March denoted by $\mathrm{F}-\mathrm{W}$ (fall, winter). The initial stratification of NOAA-7 data was by day (1430 local time) and night (0230 local time). With these initial stratifications, a check was made on the utility of the regression approach, as opposed to straightforward application of sounding temperatures to obtain the shelter temperature.

Table 6 summarizes the explained variance and the standard deviation of residuals for shelter temperatures estimated based on regression, the extrapolated temperature $T_{E}$, and the skin temperature $T_{\mathrm{SK}}$. It is apparent from Table 6 that the regression approach gives superior results to direct estimates based on retrieved temperatures. Explained variances are always highest and the standard deviations of residuals always lowest (by $0.64-1.86 \mathrm{~K}$ ) than for direct estimates.

\section{b. NOAA-6 and NOAA-7 regression statistics}

Results from the regression analyses over the course of a year for both NOAA-6 and NOAA-7 satellites are summarized in Table 7 . For each substratification of data, the three independent variables are listed in order of significance of their contribution to the estimation. Each season (S-S or F-W) of NOAA6 data was subdivided according to whether or not the skin temperature was colder than the lowest retrieval atmospheric temperature. This subdivision permits distinction of pronounced differences in the low-level atmospheric temperature structure.

TABLE 6. Explained variance $R^{2}$ and standard deviation of residuals $\sigma_{R}$ for shelter temperature estimates based on regression analysis or directly on retrieved temperatures.

\begin{tabular}{lll}
\hline A NOAA-6, spring-summer, & $N=1517$ \\
B NOAA-6, fall-winter, & $N=1099$ \\
C NOAA-7, day (year), & $N=1273$ \\
D NOAA-7, night (year), & $N=768$
\end{tabular}

\begin{tabular}{ccccc} 
Class & Statistics & Regression & $T_{E}$ & $T_{\mathrm{SK}}$ \\
\hline \multirow{2}{*}{$\mathrm{A}$} & $R^{2}:$ & 0.97 & 0.91 & 0.81 \\
& $\sigma_{R}:$ & 1.76 & 2.39 & 3.28 \\
$\mathrm{~B}$ & $R^{2}:$ & 0.93 & 0.89 & 0.91 \\
& $\sigma_{R}:$ & 2.55 & 3.61 & 3.31 \\
$\mathrm{C}$ & $R^{2}:$ & 0.95 & 0.94 & 0.85 \\
& $\sigma_{R}:$ & 2.38 & 5.26 & 4.23 \\
$\mathrm{D}$ & $R^{2}:$ & 0.94 & 0.90 & 0.93 \\
& $\sigma_{R}:$ & 2.46 & 4.70 & 3.24 \\
\hline
\end{tabular}


TABLE 7. Regression estimation statistics from extended applications and subdivisions of NOAA- 6 and NOAA-7 data. Parentheses indicate statistically insignificant predictors.

\begin{tabular}{lccccc}
\hline \multicolumn{1}{c}{ Category } & $N$ & $R^{2}$ & $\sigma_{R}$ & $\sigma_{\mathrm{SH}}$ & $\begin{array}{c}\text { Ranking of } \\
\text { predictors }\end{array}$ \\
\hline NOAA-6 & & & & & \\
S-S, $\Delta T$ pos & 754 & 0.92 & 1.68 & 1.43 & $T_{E}, M_{2},\left(T_{\mathrm{SK}}\right)$ \\
S-S, $\Delta T$ neg & 763 & 0.93 & 1.76 & 1.56 & $T_{E}, T_{\mathrm{SK}}, M_{2}$ \\
F-W, $\Delta T$ pos & 168 & 0.94 & 2.61 & 1.73 & $M_{2}, T_{E},\left(T_{\mathrm{SK}}\right)$ \\
F-W, $\Delta T$ neg & 931 & 0.93 & 2.42 & 1.85 & $T_{\mathrm{SK}}, T_{E},\left(M_{2}\right)$ \\
NOAA-7 & & & & & \\
DAY, S-S & 693 & 0.90 & 2.08 & 1.51 & $T_{E},\left(M_{2}\right),\left(T_{\mathrm{SK}}\right)$ \\
DAY, F-W & 580 & 0.95 & 2.33 & 1.77 & $T_{\mathrm{SK}}, T_{E}, M_{2}$ \\
NIGHT, $\Delta T>-4.3$ & 392 & 0.94 & 2.54 & 1.71 & $M_{2}, T_{E},\left(T_{\mathrm{SK}}\right)$ \\
NIGHT, $\Delta T \leqslant-4.3$ & 376 & 0.96 & 1.74 & 1.94 & $T_{\mathrm{SK}},\left(M_{2}\right),\left(T_{E}\right)$ \\
\hline
\end{tabular}

Day and night data from ascending and descending swaths of NOAA-7 were subdivided differently. Daytime data were separated into the 6-month springsummer and fall-winter seasons. At night, with the skin temperature almost always less than the lowest level retrieval temperature, a separation was based on the magnitude of $\Delta T$. For the smallest $\Delta T$ values, the skin temperature dominates the regression, with a significant reduction in the standard deviation of residuals. The microwave channel-2 temperature, which responds to the temperature of the lower troposphere, contributes most significantly for other nighttime conditions. The standard errors were found to be larger during individual winter months and smaller during summer months, as expected from consideration of radiation balance and heat transport mechanisms.

\section{Conclusions}

This study has demonstrated that the NESS operational temperature soundings are useful as predictors of shelter temperature. From a data set matching hourly surface observations and the operational sounding data, multiple linear regression was successfully used to relate the retrieved base atmospheric temperature, the skin temperature, and the microwave channel-2 temperature to the observed shelter temperatures. For clear and partly cloudy retrievals, which amounted to about $83 \%$ of the total, shelter temperature estimates were derived with standard errors ranging between about 1.6 and $2.6 \mathrm{~K}$. NOAA6 data were stratified by season (spring-summer and fall-winter) and by the difference between skin and retrieved air temperatures at the surface. NOAA-7 data were stratified by day and night, with daytime subdivided by season and nighttime subdivided by the skin-air temperature difference. Microwave (cloudy) retrievals were not as accurate. Best results were obtained for the 1981 spring-summer NOAA6 data with a standard error of $\sim 2.8 \mathrm{~K}$. At other seasons, the standard error rose to $\sim 4.0 \mathrm{~K}$.

For clear and partly cloudy cases, the error as represented by $\sigma_{R}$ is smaller in the estimated shelter temperatures by $\sim 1.0 \mathrm{~K}$ than has been reported for the lower levels of the soundings themselves. Root-meansquare temperature differences against radiosondes range from 2.7 to $4.0 \mathrm{~K}$ when the radiosondes are within $1.0^{\circ}$ of latitude and longitude and $\pm 12 \mathrm{~h}$ of the satellite sounding (Phillips et al., 1979; Gruber and Watkins, 1982). Since it is unlikely that the shelter temperatures estimated using satellite-derived temperature as predictors could be more accurate than the predictors themselves, we suggest that the satellite temperature soundings near the surface are somewhat more accurate than generally believed. The coincident shelter temperature data used in the matchups are closer to the sounding time than the radiosonde observations, and with five or more ground stations within $1.5^{\circ}$ latitude and longitude of the retrieval, much of the spatial variance is removed by averaging. A time-coincident and sufficiently dense radiosonde-satellite sounding data set would show the soundings to be more accurate than previous studies indicate.

Acknowledgments. Many useful discussions were held during the course of this study with Drs. Charles Dean and Larry McMillin. We also wish to thank Ms. Lynne Marquess for her support in data analysis and computer programming. This work was supported under NESS AgRISTARS Contract NA-80SAC-00749.

\section{REFERENCES}

Gruber, A., and C. D. Watkins, 1982: Statistical assessment of the quality of TIROS-N and NOAA-6 satellite soundings. Mon. Wea. Rev., 110, 867-876.

Idso, S. B., R. D. Jackson and R. J. Reginato, 1977: Remote sensing of crop yields. Science, 196, 19-25.

Jackson, R. D., S. B. Idso, R. J. Reginato and P. J. Pinter, Jr., 1981: Water Resour. Res., 12, 1133-1138.

McMillin, L. M., 1978: An improved technique for obtaining clear radiances from cloud-contaminated radiances. Mon. Wea. Rev., 106, 1590-1597.

- , and C. Dean, 1982: Evaluation of a new operational technique for producing clear radiances. $J$. Appl. Meteor., 21, 1006-1014.

Phillips, N., L. McMillin, A. Gruber and D. Wark, 1979: An evaluation of early operational temperature soundings from TIROS-N. Bull. Amer. Meteor. Soc., 60, 1188-1197.

Smith, W. L., H. M. Woolf, C. M. Hayden, D. Q. Wark and L. M. McMillin, 1979: The TIROS-N operational vertical sounder. Bull. Amer. Meteor. Soc., 60, 1177-1187.

Werbowetzki, A., 1981: Atmospheric sounding user's guide. NOAA Tech. Rep. NESS 83, 82 pp. 